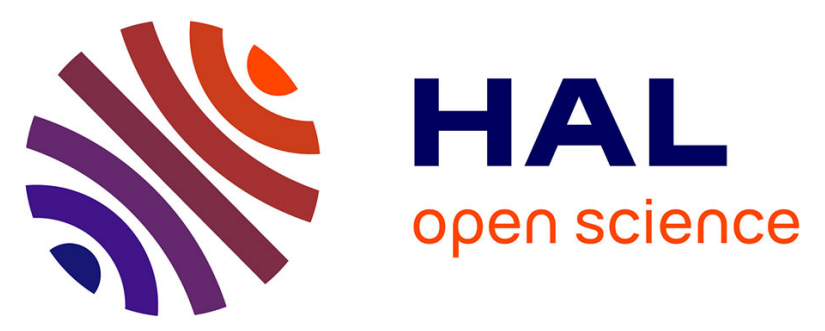

\title{
Continuous and Unconstrained Vital Signs Monitoring with Ballistocardiogram Sensors in Headrest Position
} Ibrahim Sadek, Jit Biswas, Bessam Abdulrazak, Zhang Haihong, Mounir Mokhtari

\section{- To cite this version:}

Ibrahim Sadek, Jit Biswas, Bessam Abdulrazak, Zhang Haihong, Mounir Mokhtari. Continuous and Unconstrained Vital Signs Monitoring with Ballistocardiogram Sensors in Headrest Position. IEEEEMBS International Conferences on Biomedical and Health Informatics, Feb 2017, Orlando, Florida, United States. 10.1109/BHI.2017.7897262 . hal-01450414

\author{
HAL Id: hal-01450414 \\ https://hal.science/hal-01450414
}

Submitted on 31 Jan 2017

HAL is a multi-disciplinary open access archive for the deposit and dissemination of scientific research documents, whether they are published or not. The documents may come from teaching and research institutions in France or abroad, or from public or private research centers.
L'archive ouverte pluridisciplinaire HAL, est destinée au dépôt et à la diffusion de documents scientifiques de niveau recherche, publiés ou non, émanant des établissements d'enseignement et de recherche français ou étrangers, des laboratoires publics ou privés. 


\title{
Continuous and Unconstrained Vital Signs Monitoring with Ballistocardiogram Sensors in Headrest Position
}

\author{
Ibrahim Sadek, Member, IEEE, Jit Biswas, Bessam Abdulrazak, Zhang Haihong, Mounir Mokhtari
}

\begin{abstract}
Unobtrusive and long-term monitoring of human vital signs are essential requirements for early diagnosis and prophylaxis due to many reasons, one of the most important being improving the quality of life. Currently, vital signs are continuously monitored through sensors attached to the body, such as multiple electrodes for measuring electrical activity of the heart. Such methods may be undesirable, especially for elderly, infants and other groups of people. In this paper, we introduce an improved technique for measuring heart rate from noisy ballistocardiogram signals acquired from 50 human volunteers in a sitting position using a massage chair. The signals are unobtrusively collected from a microbend fiber optic sensor embedded within the headrest of the chair, and then transmitted to a computer through a Bluetooth connection. The heart rate is computed using the multiresolution analysis of the maximal overlap discrete wavelet transform. The error between the proposed method and the reference ECG is estimated in beats per minute using the mean absolute error, where the system achieved relatively good results $(7.31 \pm 1.60)$ despite the large amount of motion artifacts produced owing to the frequent body movements and/or vibrations of the massage chair during stress relief massage. Unlike the complete ensemble empirical mode decomposition algorithm, previously employed for heart rate estimation, the suggested system is much faster. Hence, it can be used in real-time applications.
\end{abstract}

\section{INTRODUCTION}

Unobtrusive monitoring of vital signs is an increasing requirement for the medical community due to a number of reasons that may be outlined as follows: a) increased need for the recording of vital signs continuously under normal life conditions, i.e., for days and weeks, which is not achievable in hospitals; b) increased numbers of residents at assisted living facilities and numbers of elderly living alone in their own homes, caused by rapid increases in the aging population with need to self-monitor vital signs; c) the increased number of people with chronic diseases, giving rise to a need for self and remote monitoring of vital signs [1]; d) increased need for health care providers to accurately measure vital signs of their clients; e) the issue of rising health care costs

Ibrahim Sadek is with Image and Pervasive Access Laboratory (IPAL), CNRS UMI 2955, Singapore, Télécom ParisTech, Institut Mines Télécom, France, Institute for Infocomm Research $\left(\mathrm{I}^{2} \mathrm{R}\right)$, Singapore (email: stuisei2r-astar.edu.sg)

Jit Biswas is with Information Systems Technology and Design (ISTD), Singapore University of Technology and Design, Singapore (email: jit_biswasesutd.edu.sg)

Bessam Abdulrazak is with Department of Computer Science, Facult des sciences, Universit de Sherbrooke (UdeS), Canada (email: Bessam.Abdulrazak@usherbrooke.ca)

Zhang Haihong is with Institute for Infocomm Research $\left(I^{2} R\right)$, Singapore (email: hhzhangei2r.a-star.edu.sg)

Mounir Mokhtari is with Image and Pervasive Access Laboratory (IPAL), CNRS UMI 2955, Singapore, Institut Mines Télécom, France (email: mounir.mokhtari@mines-telecom.fr) throughout the world [2]. As an example, an elderly person may have the condition of sleep-disordered breathing (SDB). The SDB, better known as the obstructive sleep apnea (OSA) syndrome and associated cardiovascular complications are one of the most common clinical disorders; OSA is expected to influence approximately $14 \%$ of men and $5 \%$ of women in the general population [3].

The gold-standard approach to diagnose OSA is known as polysomnography (PSG), the PSG test should be performed in a specialist sleep clinic for a complete overnight. In principle, the sleep test includes many sensors placed on the patient body to record specific body functions such as the heart's electrical activity, brain's electrical activity, breathing, thoracoabdominal movement, blood oxygen saturation, body movement, etc. The PSG system provides accurate and real-time data. However, it imposes many limitations, i.e., complexity, invasiveness, high cost, and absence of privacy. Advanced development in hardware technology and signal processing tools enables noninvasive and unobtrusive sensing of vital signs. An alternative approach which may help diagnose OSA and other cardiovascular diseases, is the ballistocardiography.

The ballistocardiogram (BCG) signal records the mechanical activity originating from the rebound of the body, generated when the blood is pumped out of ventricles into the large blood vessel synchronous with each heart beat. BCG sensors such as the microbend fiber, may be embedded in ambient locations such as mattresses, pillows, chairs, beds, or even weighing scales [4], in order to measure BCG. Some common BCG sensing technologies in literature are microbend fiber optic sensors, fiber Bragg grating sensors, piezoresistive fabric sensors, electromechanical film and polyvinylidenefluoride film based sensors [2], [4]. One of the main advantages of the microbend fiber is that due to its immunity to electrical and electromagnetic interference it is a very suitable tool to be implemented in the magnetic resonance imaging environment.

Other advantages include small size, light weight, and lower price. Additionally, the sensor is an appropriate choice for unconstrained continuous monitoring of vital signs as it is highly sensitive to pressure changes induced due to the ballistic forces on the heart, while not required to be in close contact with the body.

The contribution of the current paper is to measure interbeat intervals (IBIs) from human subjects resting in a massage chair through a microbend fiber optic sensor (FOS) embedded in the headrest of the chair by utilizing the maximal overlap discrete wavelet transform (MODWT). 


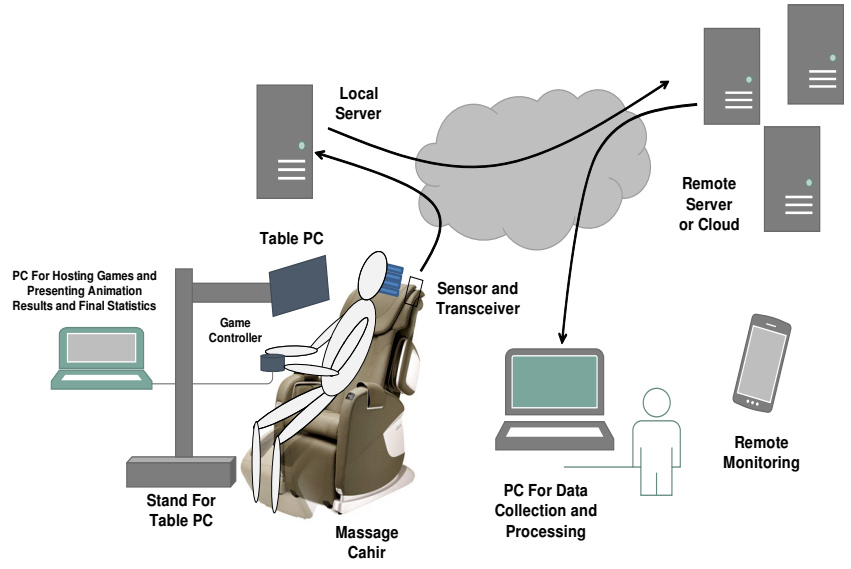

Fig. 1. Unobtrusive remote monitoring of vital signs.

Fig. 1 emphasizes how the FOS is used in a wellness application for the unobtrusive monitoring of vital signs.

\section{RELATED WORK}

In healthy subjects, the electrocardiogram (ECG) signal, which is considered as a reference to measure IBIs has a uniform and repeated template known as PQRST complex, i.e., cardiac cycle. Thus, this property facilitates the measurement of the subject's heart rate. Unlike ECG signals, the BCG morphology varies between and within subjects, and the shape of the signal is highly dependent on the subject's positions, i.e., sleeping or sitting. In addition, the raw signal is noisy and non-stationary owing to body movement, induced respiratory efforts, and the characteristics of the sensing system itself. Therefore, estimating IBIs from BCG signals is a troublesome procedure.

In [5] a translation-invariant adaptive discrete wavelet transform (DWT) is proposed to denoise BCG signals. Then, the heart rate (HR) is computed using a pseudo-period detection approach. In [6] DWT is implemented to cancel related BCG artifacts and a template matching for IBIs detection. The BCG data is collected from five healthy subjects in a sitting position using an electromechanical film sensor.

In [7] continuous wavelet transform (CWT) is introduced for HR and respiratory rate measurements, where in each case, the scale which matches the periodicity of the signal is selected. The proposed approach is applied to six healthy subjects standing on a bathroom scale equipped with multiple strain gages. A similar approach is proposed in [8], where authors used CWT with splines for optimal scale selection. However, this approach required a training phase for parameter initialization. The BCG data is acquired from seven seated healthy subjects via a piezoelectric sensor. We implemented multiresolution analysis decomposition using MODWT since the MODWT overcomes the time variant drawback of the classical DWT by upsampling the filter coefficients [9].

\section{METHODOLOGY}

\section{A. Experimental Setup and Data Collection}

The configuration of the microbend FOS implemented in our experiment is shown in Fig. 2. The system encompasses a mat, which is embedded with microbend FOS and a transceiver. The FOS data is transmitted wirelessly to a computer through a Bluetooth connection with a sampling frequency of $50 \mathrm{~Hz}$. If a pressure force is introduced to the sensing mat, the separation between the two microbenders (tooth blocks) changes. Hence, the sinusoidal amplitude of the squeezed multimode optical fiber alternates due to the ballistic forces on the heart. This ballistic force modulates the intensity of the light passing through the fiber and this modulation is subsequently recovered as a ballistocardiogram signal.

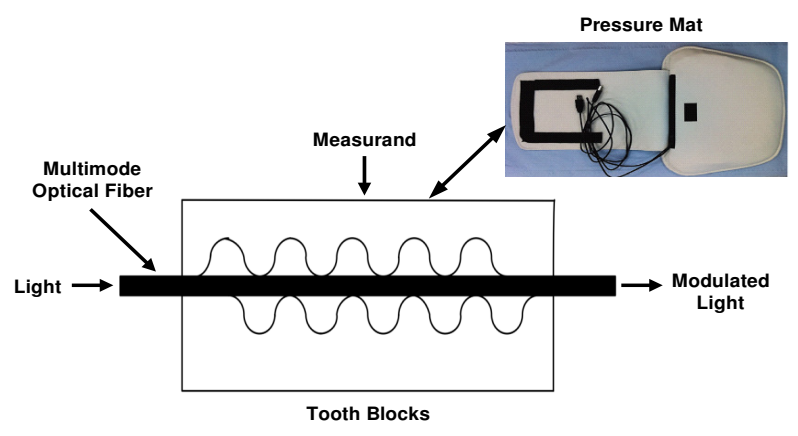

Fig. 2. Basic microbend fiber optic sensor.

The BCG signals are unobtrusively acquired from a group of 50 healthy subjects resting in a massage chair, while the sensor is embedded in the headrest of the chair. During data acquisition, subjects are instructed to complete predefined sessions, i.e., answering questionnaires, rest (no movement), and stress relief massage. At the same time, ECG signals are also collected to appraise the system performance in the HR estimation. Fig. 3 shows an example of a BCG signal with its ECG reference.
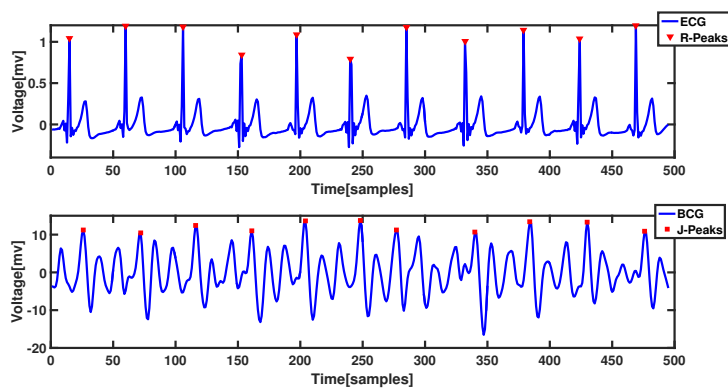

Fig. 3. BCG signal with its reference ECG signal, J-Peaks (dominant BCG peaks) represent heart beats as similar to R-Peaks in ECG.

Since subjects are performing multiple tasks, there are different scenarios for BCG data acquisition, i.e., good signals, noisy signals, and no signals. In the first, the subject is sitting still during the rest period. In the second, during the massage session, the BCG signal is strongly affected by the chair vibration. At last, the contact between the sensor mat and the subject head might be lost while the subject is filling in the questionnaire.

\section{B. Hardware Description}

The introduced algorithm is implemented using MATLAB (R2015b, MathWorks Inc., MA) on a windows based laptop 
platform (Intel core i5-4200U, 2.4GHz and 6GB RAM).

\section{Maximal Overlap Discrete Wavelet Transform}

Discrete Wavelet transform analysis is an adequate tool for transforming a time-domain signal into a time-frequencydomain signal and contrariwise. The maximal overlap discrete wavelet transform shares some common characteristics with the DWT, i.e., at each stage the input signal is convolved with a series of low-pass and high-pass filters. However, it omits the decimation of wavelet coefficients. Although the MODWT does not provide an orthogonal decomposition, it has some advantages over the DWT. For example, it is a translation-invariant approach and can manipulate any sample size n, which is not necessary to be divisible by two. Moreover, the detail and smooth coefficients of the MODWT are affiliated with zero-phase filters. Hence, it is applicable to align features in a multiresolution analysis in a meaningful way along with the original time series [9].

The MODWT decomposes a finite time signal $U_{t}$ into wavelet coefficients $\left(W_{k, t}\right)$ and approximation coefficients $\left(V_{k, t}\right) ; k$ denotes the decomposition level, and $t$ denotes the time, where $k=\{1, \ldots, K\}, t=\{1, \ldots, N-1\}, K$ represents the number of scales, and $N$ denotes the number of time points.

The MODWT wavelet $\left(W_{k, t}\right)$ and approximation $\left(V_{k, t}\right)$ coefficients for a signal $U_{t}$ including $N$ time points are derived as detailed below:

$$
\begin{aligned}
W_{k, t} & =\sum_{l=0}^{L_{k}-1} h_{k, l} \cdot U_{t-\operatorname{lmod} N} \\
V_{k, t} & =\sum_{l=0}^{L_{k}-1} g_{k, l} \cdot U_{t-\operatorname{lmod} N}
\end{aligned}
$$

The length of equivalent MODWT wavelet $\left(h_{k, l}\right)$, and approximation $\left(g_{k, l}\right)$ filters are defined such as:

$$
L_{k}=\left(2^{k}-1\right)(L-1)+1
$$

\section{Heart Rate Detection}

A sliding window of a length (10 seconds/500 samples) is used to compute the HR for BCG and ECG signals. The proposed system is presented in Fig. 4 and can be elaborated as discussed below:

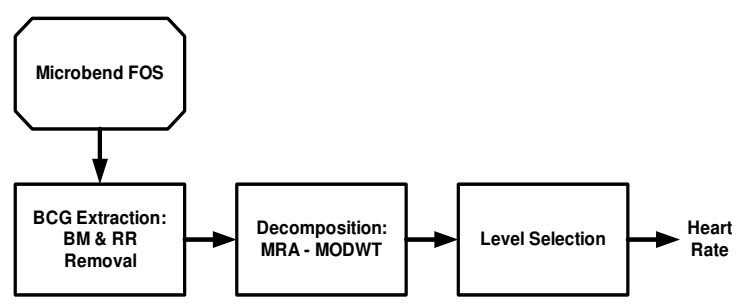

Fig. 4. The flowchart of the proposed HR estimation method; BM: Body Movement, RR: Respiratory Rate, and MRA: Multiresolution Analysis.

1) BCG Signal Extraction: The raw data of the microbend sensor consists of multiple components, i.e., BCG signals, respiratory signals, and body movements. Body movements are eliminated using a moving window of a size 1 second/50 samples with $50 \%$ overlap. Thereafter, for each window if the difference between the maximum and minimum (range) is greater than a predetermined threshold, the current window is discarded. The BCG signal is retrieved by employing a bandpass Butterworth filter with frequency limits between $1 \mathrm{~Hz}$ and $10 \mathrm{~Hz}$.

2) Decomposition: For each 10 seconds of the BCG signal, a multiresolution analysis based on MODWT is applied to decompose the signal into approximation and detail coefficients.

3) Suitable Level Selection: The $4^{\text {th }}$ level smooth coefficient is preferred for HR computation because the periodicity of the maxima well matches with the heart beats as presented in Fig. 5.
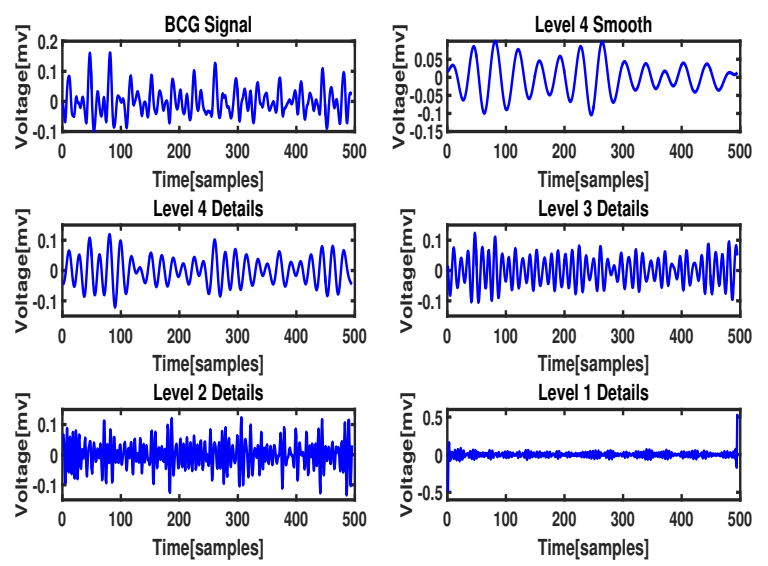

Fig. 5. Symlet-8 MODWT multiresolution decomposition of a BCG signal.

\section{RESUlTS AND Discussion}

In our study, a MODWT with level four, and $8^{\text {th }}$ order Symlets (Symlet-8) is applied to a BCG time series of 10 seconds to compute the HR. The Symlet- 8 is selected for the decomposition as discussed in [5], because the outline of the scaling function is similar to the profile of the BCG signal. The HR is calculated in beats per minute (BPM) for the two-time series. The mean and standard deviation (Std) of the mean absolute error (MAE) between ECG and BCG signals are computed across all subjects to check the quality of the proposed approach with regard to HR detection.

The work proposed in [10], [11] suggested to utilize the complete ensemble empirical mode decomposition with an adaptive noise (CEEMDAN) method to decompose the BCG signal into amplitude modulated - frequency modulated functions known as intrinsic mode functions (IMFs). Then, selecting the IMF that best represents the heart beats.

The results were promising since the CEEMDAN algorithm was able to overcome the limitations of the original empirical mode decomposition (EMD) algorithm such as end effects and mode mixing. A comparison between the CEEMDAN algorithm and the proposed MODWT multiresolution analysis is implemented to examine the performance of each method. 
TABLE I

THE MEAN AND STD OF ERROR FOR MODWT AND CEEMDAN, AND

THE RUN TIME OVER 10 SECONDS SIGNAL.

\begin{tabular}{cccc}
\hline & MAE (Average) & MAE (Std) & Run Time (Sec) \\
\hline MODWT & 7.31 & 1.60 & 0.04 \\
\hline CEEMDAN & 6.81 & 1.15 & 20 \\
\hline
\end{tabular}

The CEEMDAN is applied with a noise standard deviation of 0.35 , an ensemble size of 100 , and a maximum number of siftings of 30 . Table I presents the mean and Std of the error for both methods as well as the run time of 10 seconds signal. In addition, Fig. 6 shows box plots of the average MAE for MODWT and CEEMDAN methods. Even though the error of the CEEMDAN algorithm $(6.81 \pm 1.15)$ is slightly lower than the error for the MODWT $(7.31 \pm 1.60)$, the MODWT is much faster than CEEMDAN algorithm. Subsequently, the MODWT is more suitable for real-time applications.

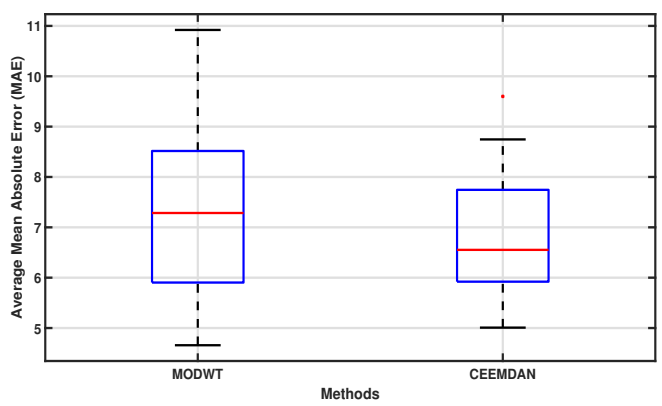

Fig. 6. Box plots of the average MAE for both CEEMDAN and MODWT methods.

In many situations, the MODWT method is able to handle the motion artifacts originated during the massage session. As shown in Fig. 7, the algorithm managed to detect all the corresponding J-Peaks in the noisy BCG signal. On the other hand, sometimes the MODWT detects more / less peaks than the reference ECG, which is not taken into account for HR estimation. Usually, this situation arises because of the induced motion artifacts. Furthermore, during the massage period the ECG electrodes might not be attached correctly to the subject body. As a result, incorrect synchronization between both signals occurred.

To sum up, in spite of the unstable environment employed to collect BCG signals, the MODWT managed to deal with motion artifacts caused by body movements and vibrations of the massage chair during relief therapy with reasonably good results.

\section{CONClusion}

In this study, a new approach is developed to measure human vital signs using BCG signals produced by a microbend FOS pressure mat embedded in the headrest of a massage chair. The proposed system is completely noninvasive and unobtrusive, where no electrodes are attached to the human body and the data can be transmitted to a personal computer via a Bluetooth connection or to a server through Wi-Fi. The HR is computed using multiresolution analysis based

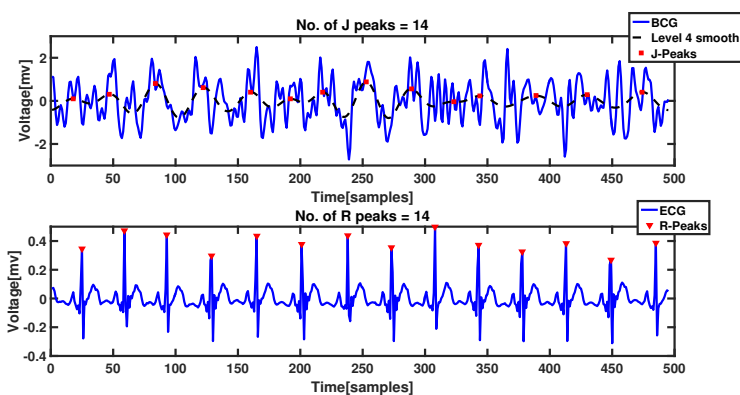

Fig. 7. BCG signal with the $4^{\text {th }}$ level smooth coefficient during a massage session.

on MODWT, where the smooth coefficient of the wavelet decomposition is chosen to compute the HR, since the periodicity of the local maxima matches with the heart beats. The MODWT attains relatively good results against the reference ECG despite the many challenges related to motion artifacts. The MODWT is much faster than the CEEMDAN algorithm. Thus, it is an appropriate tool for real-time vital signs monitoring.

\section{REFERENCES}

[1] G. Shafiq, S. Tatinati, W. T. Ang, and K. C. Veluvolu, "Automatic identification of systolic time intervals in seismocardiogram," Scientific Reports, vol. 6, p. 37524, 2016.

[2] Y. L. Zheng, X. R. Ding, C. C. Y. Poon, B. P. L. Lo, H. Zhang, X. L. Zhou, G. Z. Yang, N. Zhao, and Y. T. Zhang, "Unobtrusive sensing and wearable devices for health informatics," IEEE Transactions on Biomedical Engineering, vol. 61, no. 5, pp. 1538-1554, May 2014.

[3] R. J. Kimoff, "88 - obstructive sleep apnea," in Murray and Nadel's Textbook of Respiratory Medicine (Sixth Edition), V. C. Broaddus, R. J. Mason, J. D. Ernst, T. E. King, S. C. Lazarus, J. F. Murray, J. A. Nadel, A. S. Slutsky, and M. B. Gotway, Eds. Philadelphia: W.B. Saunders, 2016, pp. 1552 - 1568.e9.

[4] O. T. Inan, P. F. Migeotte, K. S. Park, M. Etemadi, K. Tavakolian, R. Casanella, J. Zanetti, J. Tank, I. Funtova, G. K. Prisk, and M. D. Rienzo, "Ballistocardiography and seismocardiography: A review of recent advances," IEEE Journal of Biomedical and Health Informatics, vol. 19, no. 4, pp. 1414-1427, July 2015.

[5] J. Jin, X. Wang, S. Li, and Y. Wu, "A novel heart rate detection algorithm in ballistocardiogram based on wavelet transform," in Knowledge Discovery and Data Mining, 2009. WKDD 2009. Second International Workshop on, Jan 2009, pp. 76-79.

[6] Y.-H. Noh, S.-Y. Ye, and D.-U. Jeong, "Development of the bcg feature extraction methods for unconstrained heart monitoring," in Computer Sciences and Convergence Information Technology (ICCIT), 2010 5th International Conference on, Nov 2010, pp. 923-928.

[7] S. Gilaberte, J. Gómez-Clapers, R. Casanella, and R. Pallas-Areny, "Heart and respiratory rate detection on a bathroom scale based on the ballistocardiogram and the continuous wavelet transform," in 2010 Annual International Conference of the IEEE Engineering in Medicine and Biology, Aug 2010, pp. 2557-2560.

[8] C. Alvarado-Serrano, P. S. Luna-Lozano, and R. P. As Areny, "An algorithm for beat-to-beat heart rate detection from the bcg based on the continuous spline wavelet transform," Biomedical Signal Processing and Control, vol. 27, pp. 96 - 102, 2016.

[9] D. B. Percival and A. T. Walden, Wavelet methods for time series analysis. Cambridge university press, 2006, vol. 4.

[10] I. Sadek, J. Biswas, V. F. S. Fook, and M. Mokhtari, "Automatic heart rate detection from fbg sensors using sensor fusion and enhanced empirical mode decomposition," in 2015 IEEE International Symposium on Signal Processing and Information Technology (ISSPIT), Dec 2015, pp. 349-353.

[11] I. Sadek, J. Biswas, Z. Yongwei, Z. Haihong, J. Maniyeri, C. Zhihao, T. J. Teng, N. S. Huat, and M. Mokhtari, "Sensor data quality processing for vital signs with opportunistic ambient sensing," in 2016 38th Annual International Conference of the IEEE Engineering in Medicine and Biology Society (EMBC), Aug 2016, pp. 2484-2487. 